\title{
Se actualizó la revisión de la medicación oral en diabetes tipo II ¿Qué cambió?
}

Review on oral medication in type II diabetes was updated. What did change?

Karmali K y col. Cochrane Database Syst Rev. 2017, Issue 3. CD006887

\section{Objetivos}

Comparar la efectividad y seguridad de los tratamientos de monoterapia y los combinados a base de metformina para la diabetes tipo 2 (DM2) en adultos.

\section{Fuentes de datos y selección de estudios}

Se realizó la búsqueda en MEDLINE, Embase y el Registro Central Cochrane de Ensayos Controlados (CENTRAL) de artículos en inglés utilizando los parámetros de búsqueda desarrollados en la revisión previa (2011) ${ }^{1}$; y en Drugs@FDA y ClinicalTrials.gov para evidencia no publicada. Inicialmente se realizó la búsqueda de abril de 2009 a abril de 2015, pero luego se prolongó en MEDLINE hasta diciembre de 2015.

Se incluyeron estudios que evaluaran los resultados intermedios, clínicos y de seguridad para la monoterapia (metformina, sulfonilureas, tiazolidindionas, inhibidores de dipeptidil peptidasa-4
[DPP-4], agonistas del péptido-1 [GLP-1] tipo glucagón, e inhibidores del cotransportador-2 de la glucosa sódica [SGLT-2]), o la terapia combinada basada en metformina (más uno de estos fármacos o insulina).

Dos revisores independientes evaluaron los resultados clínicos e intermedios y la seguridad de la monoterapia versus tratamientos combinados a base de metformina. Otros dos revisores extrajeron la información y evaluaron el riesgo de sesgo en forma independiente. Finalmente, dos revisores clasificaron la fuerza de la evidencia en forma secuencial utilizando el protocolo que define los criterios GRADE.

\section{Resultados Principales}

Se incluyeron 216 estudios y se encontraron pruebas de moderada o alta evidencia. A continuación se detallan los resultados principales en la tabla 1.

Tabla 1. Comparación en efectividad y seguridad de los tratamientos de monoterapia y combinados a base de metformina.

\begin{tabular}{|c|c|c|}
\hline \multirow{4}{*}{ 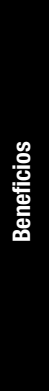 } & HbA1c & $\begin{array}{l}\text { La mayoría de las drogas o combinaciones a base de metformina reducen la HbA1C en forma similar } \\
\text { Inhibidores DPP-4 reducen menos la HbA1C que la metformina. }\end{array}$ \\
\hline & Peso & Metformina, Inhibidores DPP-4, Agonistas GLP-1 e Inhibidores SGLT-2 reducen o mantienen el peso. \\
\hline & Tensión arterial sistólica & $\begin{array}{l}\text { Inhibidores SGLT-2 y Agonistas GLP- } 1 \text { asociados a metformina reducen TAS entre } 3 \text { a } 5 \text { mmHg comparados } \\
\text { con metformina. }\end{array}$ \\
\hline & Mortalidad cardiovascular & $\begin{array}{l}\text { Las sulfonilureas presentaron un } 50 \text { a } \mathbf{7 0} \% \text { más de mortalidad cardiovascular que la metformina en estudios } \\
\text { de } 2 \text { años de duración (RRA 0,1\% a 2,9\% en ensayos clínicos controlados y aleatorizados) }\end{array}$ \\
\hline \multirow{3}{*}{ 营 } & Sulfonilureas & Aumenta el riesgo de hipoglucemias comparado con otras drogas \\
\hline & Metformina & $\begin{array}{l}\text { Mayor riesgo de efectos adversos gastrointestinales (excepto Agonistas GLP-1que aumentan el riesgo de } \\
\text { náuseas y vómitos } 1,5 \text { veces) }\end{array}$ \\
\hline & Inhibidores SGLT-2 & Aumenta el riesgo de infecciones micóticas en genitales \\
\hline
\end{tabular}

DPP-4 dipeptidil peptidasa-4; GLP-1: péptido-1 tipo glucagón; SGLT-2: cotransportador-2 de la glucosa sódica; DR: diferencia de riesgo; TAS: tensión arterial sistólica.

\section{Conclusiones}

La evidencia apoya la metformina como primera línea de tratamiento dados sus beneficios en el descenso de $\mathrm{HbA1C}$, peso y mortalidad cardiovascular (vs. sulfonilureas) y su perfil de seguridad. En la revisión se proporciona información que compara los beneficios y los daños de los medicamentos para la diabetes. Ante la ausencia de evidencia concluyente en relación a los resultados clínicos a largo plazo y de seguridad para la mayoría de las medicaciones comparadas, la evidencia sirve para facilitar las decisiones personalizadas y la toma de decisiones compartidas entre los agentes reguladores y los que abonan los tratamientos.
Fuente de financiamiento/conflicto de interés: estudio dirigido por el Centro de Práctica Basada en la Evidencia, Universidad Johns Hopkins, contratado por la Agencia para la Investigación y Calidad de los Cuidados de Salud (AHRQ). Los investigadores no refieren conflictos de interés. 


\section{Comentario}

La diabetes mellitus es una de las principales causas de morbimortalidad, siendo su forma más frecuente la DM2. Su tratamiento se basa en cambios en el estilo de vida y tratamientos farmacológicos. Actualmente se cuenta con seis grupos diferentes de tratamientos farmacológicos aprobados por la agencia reguladora de alimentos y medicamentos de los EE.UU. (FDA, por sus iniciales en inglés) y constantemente se acrecientan e investigan nuevas drogas en cada grupo. De hecho, luego de la revisión previa se incorporó un grupo nuevo de drogas (inhibidores SGLT-2), es por este motivo que era importante actualizar la revisión.

Como resultado de esta revisión, el Colegio Americano de Médicos (American College of Physicians) desarrolló una guía actualizada ${ }^{2}$ de la evidencia clínica provista para los tratamientos farmacológicos orales y ha modificado las recomendaciones principales. En su guía previa ${ }^{3}$ promovía el uso de tratamientos farmacológicos cuando los cambios en el estilo de vida eran insuficientes, y determinaba en una segunda recomendación el uso de metformina cuando no estaba contraindicada. Ante la nueva evidencia la recomendación principal ahora es que se debe prescribir metformina como tratamiento farmacológico inicial cuando sea necesario mejorar el control glucémico (recomendación fuerte; evidencia de moderada calidad). Es un cambio sutil pero que refuerza a la metformina como primera línea de tratamiento. Y en relación a la recomendación final, se refuerza la necesidad de agregar un segundo agente pero en esta nueva guía se especifica que debe pertenecer a cuatro de los cinco grupos restantes (evitar agonistas GLP-1), y que esta elección debe ser tomada en conjunto con el paciente luego de evaluar beneficios, efectos adversos y costos (recomendación débil; evidencia de moderada calidad).

\section{Conclusiones del comentador}

Si bien los estudios incluidos tenían un mínimo de tres meses de seguimiento, sólo el $4 \%$ se extiende más allá de los dos años. Esto dificulta la obtención de evidencia fuerte en relación con los resultados clínicos, más relevantes para los pacientes y a largo plazo, y permanece abierta la necesidad de trabajar en esta área dada la alta prevalencia de la enfermedad.

María Teste [Salud Comunitaria - Municipalidad de Trenque Lauquen, maryteste@gmail.com]

Teste M. Se actualizó la revisión de la medicación oral en diabetes tipo II. ¿Qué cambió? Evid Act Pract Ambul. 2018;21(1):29-30. Comentado de: Bolen $\mathrm{S}$ y col. Diabetes Medications for Adults With Type 2 Diabetes: An Update. Comparative Effectiveness Review. Agency for Healthcare Research and Quality (AHRQ) Publication No. 16-EHC013-EF April 2016. PMID: 27227214.

\section{Referencias Bibliográficas}

1. Bennett WL Maruthur NM, Singh S, Segal JB, Wilson LM, Chatterjee R, et al Comparative effectiveness and safety of medications for type 2 diabetes: an update including new drugs and 2-drug combinations. Ann Intern Med. - 2011. 154:602-13.

2. Amir Qaseem MD, PhD, MHA [et al] Oral Pharmacologic Treatment of Type 2 Diabetes Mellitus: A Clinical Practice Guideline Update From the American College of Physicians: Ann Intern Med, 2017. 166:270-290

3. Amir Qaseem MD, PhD, MHA [et al] Oral Pharmacologic Treatment of Type 2 Diabetes Mellitus: A Clinical Practice Guideline From the American College of Physicians: Ann Intern Med, 2012.156:218-231 